\title{
Earlier Initiation of Antiretroviral Treatment Coincides With an Initial Control of the HIV-1 Sub-Subtype F1 Outbreak Among Men-Having-Sex-With-Men in Flanders, Belgium
}

\section{OPEN ACCESS}

Edited by:

Joris Hemelaar,

University of Oxford, United Kingdom

Reviewed by:

Fernando Gonzalez-Candelas,

University of Valencia, Spain

Dimitrios Paraskevis,

National and Kapodistrian University

of Athens, Greece

${ }^{*}$ Correspondence:

Kristel Van Laethem

kristel.vanlaethem@uzleuven.be

Specialty section:

This article was submitted to

Virology,

a section of the journal

Frontiers in Microbiology

Received: 13 October 2018 Accepted: 11 March 2019

Published: 26 March 2019

Citation:

Vinken L, Fransen K, Cuypers L, Alexiev I, Balotta C, Debaisieux L, Seguin-Devaux C, García Ribas S,

Gomes $P$, Incardona F, Kaiser R,

Ruelle J, Sayan M, Paraschiv S, Paredes $R$, Peeters $M$, Sönnerborg $A$,

Vancutsem $E$, Vandamme $A-M$, Van den Wijngaert S, Van Ranst M, Verhofstede C, Stadler T, Lemey P and Van Laethem K (2019) Earlier Initiation of Antiretroviral Treatment

Coincides With an Initial Control of the HIV-1 Sub-Subtype F1 Outbreak Among Men-Having-Sex-With-Men

in Flanders, Belgium.

Front. Microbiol. 10:613.

doi: 10.3389/fmicb.2019.00613
Lore Vinken ${ }^{1}$, Katrien Fransen ${ }^{2}$, Lize Cuypers ${ }^{1}$, Ivailo Alexiev ${ }^{3}$, Claudia Balotta ${ }^{4}$, Laurent Debaisieux ${ }^{5}$, Carole Seguin-Devaux ${ }^{6}$, Sergio García Ribas ${ }^{2}$, Perpétua Gomes ${ }^{7,8}$, Francesca Incardona ${ }^{9}$, Rolf Kaiser ${ }^{10}$, Jean Ruelle ${ }^{11}$, Murat Sayan ${ }^{12,13}$, Simona Paraschiv ${ }^{14}$, Roger Paredes ${ }^{15}$, Martine Peeters ${ }^{16}$, Anders Sönnerborg ${ }^{17}$, Ellen Vancutsem ${ }^{18}$, Anne-Mieke Vandamme ${ }^{1,19}$, Sigi Van den Wijngaert ${ }^{20}$, Marc Van Ranst ${ }^{1,21}$, Chris Verhofstede ${ }^{22}$, Tanja Stadler ${ }^{23,24}$, Philippe Lemey ${ }^{1}$ and Kristel Van Laethem ${ }^{1,21 *}$

${ }^{1}$ Laboratory of Clinical and Epidemiological Virology, Department of Microbiology and Immunology, Rega Institute for Medical Research, KU Leuven, Leuven, Belgium, ${ }^{2}$ AIDS Reference Laboratory, Department of Clinical Sciences, Institute of Tropical Medicine Antwerp, Antwerp, Belgium, ${ }^{3}$ National Reference Confirmatory Laboratory of HIV, National Center of Infectious and Parasitic Diseases, Sofia, Bulgaria, ${ }^{4}$ Infectious Diseases and Immunopathology Section, 'L. Sacco' Department of Biomedical and Clinical Sciences, 'L. Sacco' Hospital, University of Milan, Milan, Italy, ${ }^{5}$ AIDS Reference Laboratory, CUB-Hopital Erasme, Université Libre de Bruxelles, Brussels, Belgium, ${ }^{6}$ Laboratory of Retrovirology, Department of Infection and Immunity, Luxembourg Institute of Health, Esch-sur-Alzette, Luxembourg, ' Serviço de Patologia Clínica, Laboratorio de Biologia Molecular, LMCBM, Centro Hospitalar Lisboa Ocidental, Hospital Egas Moniz, Lisbon, Portugal, ${ }^{8}$ Centro de Investigação Interdisciplinar Egas Moniz, Instituto Universitário Egas Moniz, Almada, Portugal, ${ }^{9}$ EuResist Network GEIE, Rome, Italy, ${ }^{10}$ Institute of Virology, University of Cologne, Cologne, Germany, ${ }^{11}$ Unit of Medical Microbiology, Institute of Experimental and Clinical Research, Université catholique de Louvain, Brussels, Belgium, ${ }^{12}$ PCR Unit, Clinical Laboratory, Kocaeli University, Izmit, Turkey, ${ }^{13}$ Research Center of Experimental Health Sciences, Near East University, Nicosia, Cyprus, ${ }^{14}$ Molecular Diagnostics Laboratory, National Institute for Infectious Diseases 'Matei Bals', Bucharest, Romania, ${ }^{15}$ IrsiCaixa AIDS Research Institute, Universitat Autònoma de Barcelona, Badalona, Spain, ${ }^{16}$ UMI 233 TransVIHMI/INSERM1175, Institut de Recherche pour le Développement, University of Montpellier, Montpellier, France, ${ }^{17}$ Division of Clinical Microbiology, Department of Laboratory Medicine, Karolinska Institutet, Stockholm, Sweden, ${ }^{18}$ AIDS Reference Laboratory, Department of Microbiology and Infection Control, Universitair Ziekenhuis Brussel, Brussels, Belgium, 19 Unidade de Microbiologia, Center for Global Health and Tropical Medicine, Instituto de Higiene e Medicina Tropical, Universidade Nova de Lisboa, Lisbon, Portugal, ${ }^{20}$ AIDS Reference Laboratory, Department of Microbiology, Saint-Pierre University Hospital, Brussels, Belgium, ${ }^{21}$ AIDS Reference Laboratory, University Hospitals Leuven, Leuven, Belgium, ${ }^{22}$ AIDS Reference Laboratory, Department of Clinical Chemistry, Microbiology and Immunology, Ghent University, Ghent, Belgium, ${ }^{23}$ Department of Biosystems Science and Engineering, ETH Zürich, Basel, Switzerland, ${ }^{24}$ Swiss Institute of Bioinformatics, Lausanne, Switzerland

Human immunodeficiency virus type 1 (HIV-1) non-B subtype infections occurred in Belgium since the 1980s, mainly amongst migrants and heterosexuals, whereas subtype B predominated in men-having-sex-with-men (MSM). In the last decade, the diagnosis of F1 sub-subtype in particular has increased substantially, which prompted us to perform a detailed reconstruction of its epidemiological history. To this purpose, the Belgian AIDS Reference Laboratories collected HIV-1 pol sequences from all subsubtype F1-infected patients for whom genotypic drug resistance testing was requested 
as part of routine clinical follow-up. This data was complemented with HIV-1 pol sequences from countries with a high burden of $\mathrm{F} 1$ infections or a potential role in the global origin of sub-subtype F1. The molecular epidemiology of the Belgian subtype F1 epidemic was investigated using Bayesian phylogenetic inference and transmission dynamics were characterized based on birth-death models. F1 sequences were retained from 297 patients diagnosed and linked to care in Belgium between 1988 and 2015. Phylogenetic inference indicated that among the 297 Belgian F1 sequences, 191 belonged to a monophyletic group that mainly contained sequences from people likely infected in Belgium (OR 26.67, 95\% Cl 9.59-74.15), diagnosed in Flanders (OR $7.28,95 \% \mathrm{Cl} 4.23-12.53$ ), diagnosed at a recent stage of infection (OR 7.19, 95\% Cl 2.88-17.95) or declared to be MSM (OR 34.8, 95\% Cl 16.0-75.6). Together with a Spanish clade, this Belgian clade was embedded in the genetic diversity of Brazilian subtype F1 strains and most probably emerged after one or only a few migration events from Brazil to the European continent before 2002. The origin of the Belgian outbreak was dated back to 2002 (95\% higher posterior density 2000-2004) and birth-death models suggested that its extensive growth had been controlled $\left(R_{e}<1\right)$ by 2012, coinciding with a time period where delay in antiretroviral treatment initiation substantially declined. In conclusion, phylogenetic reconstruction of the Belgian HIV-1 sub-subtype F1 epidemic illustrates the introduction and substantial dissemination of viral strains in a geographically restricted risk group that was most likely controlled by effective treatment as prevention.

Keywords: HIV-1, sub-subtype F1, Belgium, phylodynamics, men-having-sex-with-men, treatment as prevention

\section{INTRODUCTION}

Human immunodeficiency virus type 1 (HIV-1) originated after at least four separate cross-species transmission events from nonhuman primates to humans in central Africa, which resulted in four groups $(\mathrm{M}, \mathrm{N}, \mathrm{O}$, and $\mathrm{P})$ that emerged in the early twentieth century (Peeters et al., 2014). Since then, HIV-1 group M lineages have diversified and the current genetic diversity existing within the pandemic group $\mathrm{M}$ is the result of subsequent evolution and spread within the human population. Using phylogenetic analysis, HIV-1 group $M$ can be classified into nine major subtypes (A-D, F-H, J, and $\mathrm{K}$ ) and a few sub-subtypes (like F1 and F2) which reflects a combination of several founder effects and incomplete sampling of the original source population in central Africa (Rambaut et al., 2004). On the other hand, the various circulating recombinant forms (CRFs) originated from co-circulation of subtypes that previously evolved in compartmentalized epidemics.

The HIV-1 subtype B lineage that is responsible for the majority of HIV-1 infections in Europe, was first exported from central Africa to the Caribbean region around 1967 from where it was subsequently introduced in the United States and spread over to other American and European countries, mainly via networks of men-having-sex-with-men (MSM) and people who injects drugs (PWID) (Abecasis et al., 2013; Junqueira and Almeida, 2016; Magiorkinis et al., 2016; Worobey et al., 2016). HIV-1 subtype B was the predominant subtype among Belgians who were diagnosed with HIV-1 between 1985 and 1994 in Antwerp
(Belgium), although the presence of other pure subtypes such as A, C, D, F, G, and $\mathrm{H}$ already revealed an early introduction of HIV-1 non-B subtypes to the local population (Fransen et al., 1996). Nevertheless, Belgian MSM remained almost exclusively infected with subtype B strains. These findings were confirmed in an independent study where heterosexual contact, African origin and more recent diagnosis were predictors for non-B infection in HIV-1 patients diagnosed between 1983 and 2001 in two other Belgian cities (Brussels and Leuven) (Snoeck et al., 2004). Subsequent local and national surveillance studies that included HIV-1 diagnoses between 1998 and 2012 confirmed the association between subtype B infections, MSM and Caucasian origin. In contrast, HIV-1 non-B infections in Belgium, of which HIV-1 subtype A, C, and CRF02_AG infections were the most prevalent, were associated to migrants from sub-Saharan Africa and individuals showing heterosexual risk behavior (Vercauteren et al., 2008; Chalmet et al., 2010; Pineda-Peña et al., 2014).

In the last decade, sub-subtype F1 diagnoses have markedly increased among newly diagnosed HIV-1 patients linked to care in Belgium. In the light of two Spanish studies that reported lower therapy response rates in HIV-1 sub-subtype F1 infections (Pernas et al., 2014; Cid-Silva et al., 2018), we aimed to investigate the evolutionary history and distinctive socio-demographic and epidemiological features of the HIV-1 sub-subtype F1 epidemic in Belgium. The spatiotemporal origin of the ancestor of the Belgian F1 epidemic and evolutionary links among locations were determined, risk factors were identified, and transmission dynamics were quantified. 


\section{MATERIALS AND METHODS}

\section{Viral Sequences and Associated Information}

Human immunodeficiency virus type 1 sub-subtype F1 pol sequences spanning the gene fragments that encode protease (PR) and the $5^{\prime}$-end of reverse transcriptase (RT) were collected from all patients for whom drug resistance testing was requested as part of routine clinical follow-up at six AIDS reference laboratories (ARL) in Antwerp, Brussels, Ghent, and Leuven (up until March 2015). These ARL perform approximately $90 \%$ of the HIV-1 drug resistance testing in Belgium. HIV-1 sub-subtype F1 pol sequences were requested from partners in other European and African countries where circulation of F1 infections has been reported (Palma et al., 2007; Paraschiv et al., 2009; Frentz et al., 2010; Lai et al., 2012; Ivanov et al., 2013; Sayan et al., 2013; Alexiev et al., 2015). Furthermore, all available HIV-1 sub-subtype F1 pol sequences were extracted from the Los Alamos HIV Sequence Database (LANL) ${ }^{1}$ (Triques et al., 1999; Montano et al., 2005; Aulicino et al., 2007). Most viral genetic sequences were annotated with sampling time and location, which were used as calibration and discrete traits, respectively, in an analysis of spatiotemporal dispersion patterns (Supplementary Table S1). Patient socio-demographics and detailed sample information were collected from mandatory reporting and medical records at the participating ARL in Belgium (gender, year of birth, HIV-1 diagnosis date, infection stage at diagnosis, country of birth, probable country of infection, sexual risk factor for HIV1 acquisition, viral load, CD4 and CD8 count associated to the HIV-1 pol sequence, and therapy initiation date). Infection stage at diagnosis was defined based upon laboratory data: HIV-1 confirmation results with a positive p24 test combined with a negative or undetermined blot or INNO-LIA HIV I/II (Fujirebio Europe, Ghent, Belgium) were classified as acute infections while results with a positive blot lacking a p31 band as recent infections (Verhofstede et al., 2017). In all other instances, at least when records were available, infections were classified as chronic. The study was approved by the Ethical Committee Research UZ/KU Leuven (reference S58359). This study did not require written informed consent by study participants as it only used data collected within routine clinical practice, but patients who opted out of research studies were not included. The entire dataset for this manuscript is not publicly available because it represents a dense sample of our epidemic and inappropriate use could endanger the privacy of the patients. A proportional down-sampling of the dataset (30\%) is accessible via GenBank (accession numbers, MK458435MK458523). Requests to access the entire dataset combined with well-defined project proposals can be mailed to the corresponding author who will submit it to the local ethical committee for ethical and legal clearance when not in conflict with already on-going research.

\footnotetext{
${ }^{1}$ http://www.hiv.lanl.gov
}

\section{Quality Control and Compilation of Sequences}

The quality of HIV-1 sequences that were generated by the Belgian ARL was assured by quality management systems that are conform to the ISO 15189 standard. In addition, the quality of all collected HIV-1 sequences was subsequently verified using the Quality Control tool hosted on the HIV sequence database LANL. Only sequences with no evidence of hyper-mutations and a maximum of 7 stop codons and/or frameshifts were retained for further analyses. Only pure subsubtype F1 strains were retained in the study. Therefore, HIV-1 subtype classification was confirmed using the online automated subtyping tools COMET v1.0 (Struck et al., 2014) and Rega v3 (Pineda-Peña et al., 2013). In case of a non-F1 classification by COMET v1.0 or Rega v3 (7\% discordant classifications), sequences were subsequently subjected to jpHMM (Schultz et al., 2009; version March 2015). If scored as F1 or F2 sub-subtype by jpHMM, recombination was ruled out using Simplot (Lole et al., 1999). Four HIV-1 subtype B reference sequences were added to the dataset, serving as an outgroup for subsequent phylogenetic analyses (GenBank accession numbers K03455, AY423387, AY173951, and AY331295). Nucleotide sequences were aligned using Muscle v3.7 (Edgar, 2004) and manually edited in MEGA 5 (Tamura et al., 2011). Nucleotide positions encoding for 43 surveillance drug resistance mutations (Bennett et al., 2009) were removed from the alignment to avoid the effect of drug-induced convergent evolution. Duplicate sequences were identified using the ElimDupes tool (LANL) and were removed when originating from the same sample. For patients with multiple sequences, only the first sequence was retained. The final alignment contained $1866 \mathrm{HIV}-1$ sub-subtype F1 sequences trimmed to the common length of 798 nucleotide positions, spanning sites 2253 to 2549 and 2661 to 3290 (as compared to the HIV-1 subtype B reference sequence HXB2, accession number K03455) (Supplementary Table S1).

\section{Phylogenetic and Phylogeographic Analyses}

The spatiotemporal origin of the Belgian HIV-1 sub-subtype F1 epidemic was investigated using a Bayesian approach. Prior to this analysis, we examined the accumulation of divergence over the sampling time span using Tempest v1.5 (Rambaut et al., 2016) to assure that sampling dates could be used as calibration. Markov chain Monte Carlo analyses were performed using BEAST v1.8.4 in conjunction with BEAGLE to improve the computational performance (Ayres et al., 2012; Suchard et al., 2018). We specified a GTR nucleotide substitution model with discrete gamma distributed rate variation among sites. Among-lineage variation in evolutionary rates was modeled using a lognormal relaxed molecular clock and a flexible non-parametric Bayesian Skygrid was specified as a coalescent prior. The analyses were run in triplicate for 100 million states and trees were sampled every 10,000th state. Convergence and mixing properties of the Markov chain Monte Carlo chains were assessed with Tracer v1.6 (Rambaut et al., 2018). Results of several chains were combined after removal of the burn-in, and a MCC tree was summarized 
using TreeAnnotator within the BEAST package. The timed migration process was reconstructed using a model that allows for non-reversible migration rates. Trees were visualized with FigTree v1.4.3 and iTOL v4.2.3 (Letunic and Bork, 2016).

\section{Phylodynamic Analysis of the Infection Cluster Including Belgian Sequences}

Phylodynamic analyses were performed on an infection cluster that mainly contained sequences from our Belgian cohort. A birth-death model was applied to the Belgian sequences included in this infection cluster (191 sequences) in order to quantify transmission dynamics through time. The analyses were performed in BEAST v2 (Bouckaert et al., 2014) using the BDSKY add-on (Stadler et al., 2013). The molecular evolution and clock model were chosen as in the phylogeographic analysis. For the birth-death-skyline parameters, we used a $\operatorname{LogNorm}(0,1)$ prior for the effective reproductive number, a $\operatorname{LogNorm}(0,1)$ prior for the becoming-non-infectious rate in units per year (i.e., the inverse of the time duration of being infectious in unit of years), and a Unif(0,infinity) prior for the origin of the epidemic. The dimension of the effective reproductive number and the becoming-non-infectious rate was set to 10 , meaning the parameters were estimated for 10 equally spaced intervals between the origin and the most recent sample. The sampling proportion was fixed to 0.65 (and 0.8 in a second analysis) for the time between the first and last sample, and 0 prior to the first sample.

\section{Statistical Analysis}

Socio-demographic, virological and clinical variables were reported as absolute numbers, percentages and medians with their respective interquartile ranges (IQR), where appropriate. Their association with a Belgian F1 outbreak was evaluated using univariate and multivariate logistic regression in the statistical $\mathrm{R}$ software v3.5.1, with statistical significance set at $p$-value $<0.05$. For these analyses, only patients with available information for a specific variable were included.

\section{RESULTS}

\section{Increase of Subtype F1 Diagnoses in Belgium}

Up to February 2015, 297 HIV-1 sub-subtype F1 infections were newly diagnosed in the participating Belgian ARL (Figure 1), of which 137 were analyzed in Antwerp (46\%), 95 in Brussels (32\%), 38 in Leuven (13\%), and 27 in Ghent (9\%). The median age of people diagnosed with an HIV-1 sub-subtype F1 infection was 34 years (IQR 29-41) and 77\% were men. Over the course of 15 years, the number of HIV-1 sub-subtype F1 diagnoses increased from 3 in March 2001 - February 2003 to 88 in March 2013 - February 2015 (Figure 1A). Sub-subtype F1 infections were initially mainly diagnosed in women (65\%), but after February 2005 men became the most affected gender (87\%). Of the sub-subtype F1 infections for which the respective information was registered, the majority were diagnosed in people born in Belgium (59\%, 141/238) and in people who declared that they probably acquired their infection in Belgium $(82 \%, 138 / 168)$. The sub-subtype F1 infections were found in homosexual $(51 \%, 117 / 231)$, heterosexual $(35 \%, 80 / 231)$, and bisexual risk (15\%, 34/231) groups (Figure 1B).

\section{The Belgian Sub-Subtype F1 Epidemic Within a Global Context}

Bayesian phylogenetic analyses were used to estimate the geographical and temporal origin of the HIV-1 sub-subtype F1 strains in our national cohort and to investigate viral dissemination from Belgium to other countries. The final dataset included 1866 sequences that were mainly sampled in Europe (75\%) and Brazil (20\%) (Supplementary Table S1). We found sufficient temporal signal to pursue phylodynamic analysis and used the resulting empirical distribution of the dated phylogenetic trees in a subsequently phylogeographic analysis.

The phylogeographic analysis revealed the divergence of subsubtype F1 into two well-supported clades for which (i) one clade included mainly Brazilian and Portuguese strains together with a Belgian subclade, while (ii) the other predominantly Belgian, Romanian and Portuguese sequences (upper and lower clade in Figure 2, Supplementary Figure S1). Their divergence dates back 50 years ago (1968, 95\% higher posterior density (HPD) interval of 1963-1973), with an estimated start of local dissemination of HIV-1 F1 in Brazil around 1973 (95\% HPD 1969-1975) and subsequently in Portugal around 1979 (95\% HPD 1976-1981) (Figure 2 upper clade). In this upper clade (i), one large Belgian cluster, subsequently referred to as the sub-subtype F1 Belgian outbreak, is embedded within a WesternEuropean clade that in turn is nested within the larger Brazilian clade. The Belgian clade contains four sequences sampled outside Belgium (one from Brazil, one from Luxembourg, and two from Germany), while the Western-European clade consists of the Belgian clade together with three basal sequences from Switzerland, one large well-supported Spanish clade and one Romanian sequence that branches outside the Spanish and Belgian clade. The founder of the HIV-1 sub-subtype F1 strains that are currently circulating in Western-Europe, probably migrated from Brazil to Europe between 1990 and 1999 (95\% HPD 1987-2002), whereas the origin of the Belgian and Spanish outbreaks were dated back to 2002 (95\% HPD 2000-2004) and 2006 (95\% HPD 2004-2008), respectively. Brazil is estimated as the most likely geographical origin of the Western-European clade because the Western-European clade was embedded within the large Brazilian sub-subtype F1 diversity and because one strain that was sampled in Brazil and three strains that were sampled from Brazilians residing in Belgium were also positioned within the Belgian clade. Although Belgium was assigned as ancestral location state of this Western-European clade, the long interconnecting branch suggests that current sampling is insufficient to provide conclusive results as to the specific migration event(s) that finally led to the two large outbreaks in Western Europe (Belgium and Spain).

The remaining sub-subtype F1 sequences from our Belgian cohort represent single lineages or small clusters that are 
A

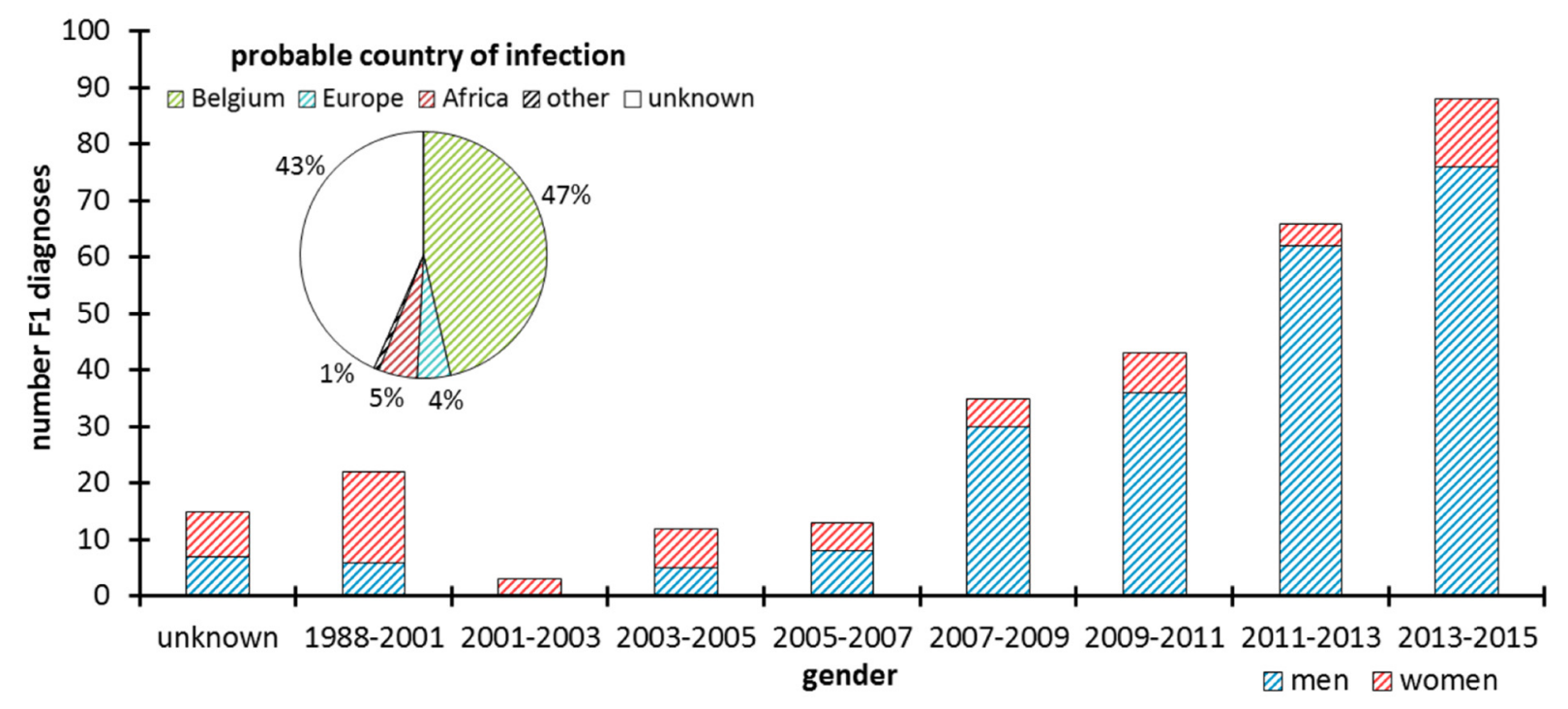

B

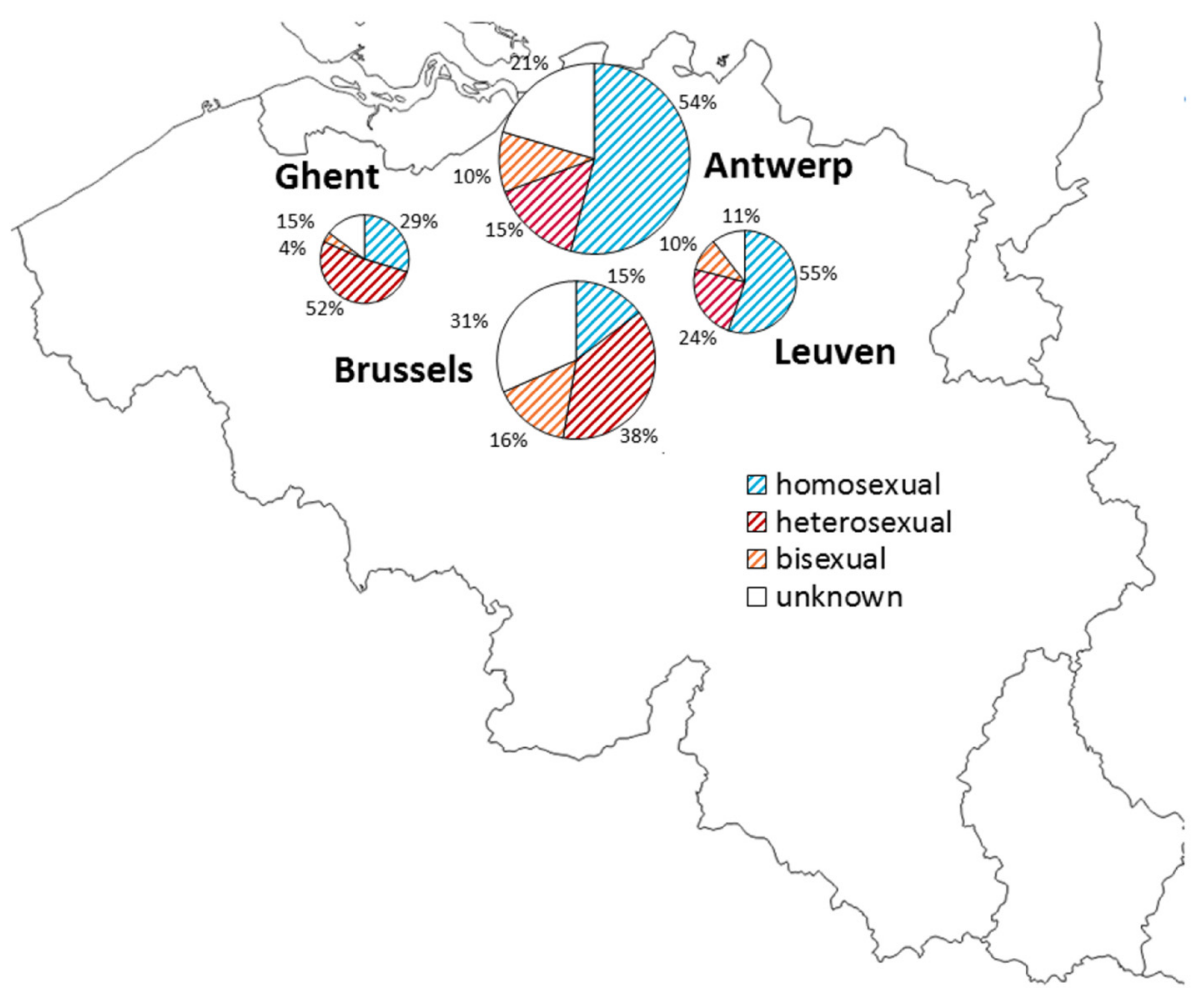

FIGURE 1 | HIV-1 sub-subtype F1 diagnoses in Belgium. Panel (A) Number of men and women diagnosed with an HIV-1 sub-subtype F1 infection according to 2-year intervals and based upon a pol-genotypic drug resistance test that was requested as part of routine clinical follow-up. The pie chart displays the proportion of people who probably acquired the HIV-1 sub-subtype F1 infection in Belgium, Europe, Africa, or another or unknown region. Panel (B) Geographic and proportional distribution of HIV-1 sub-subtype F1 diagnoses in the AIDS Reference Laboratories of Antwerp, Brussels, Leuven, and Ghent. The pie charts display the proportion of people reporting homosexual, heterosexual, or bisexual contacts as risk factor for HIV-1 infection. 


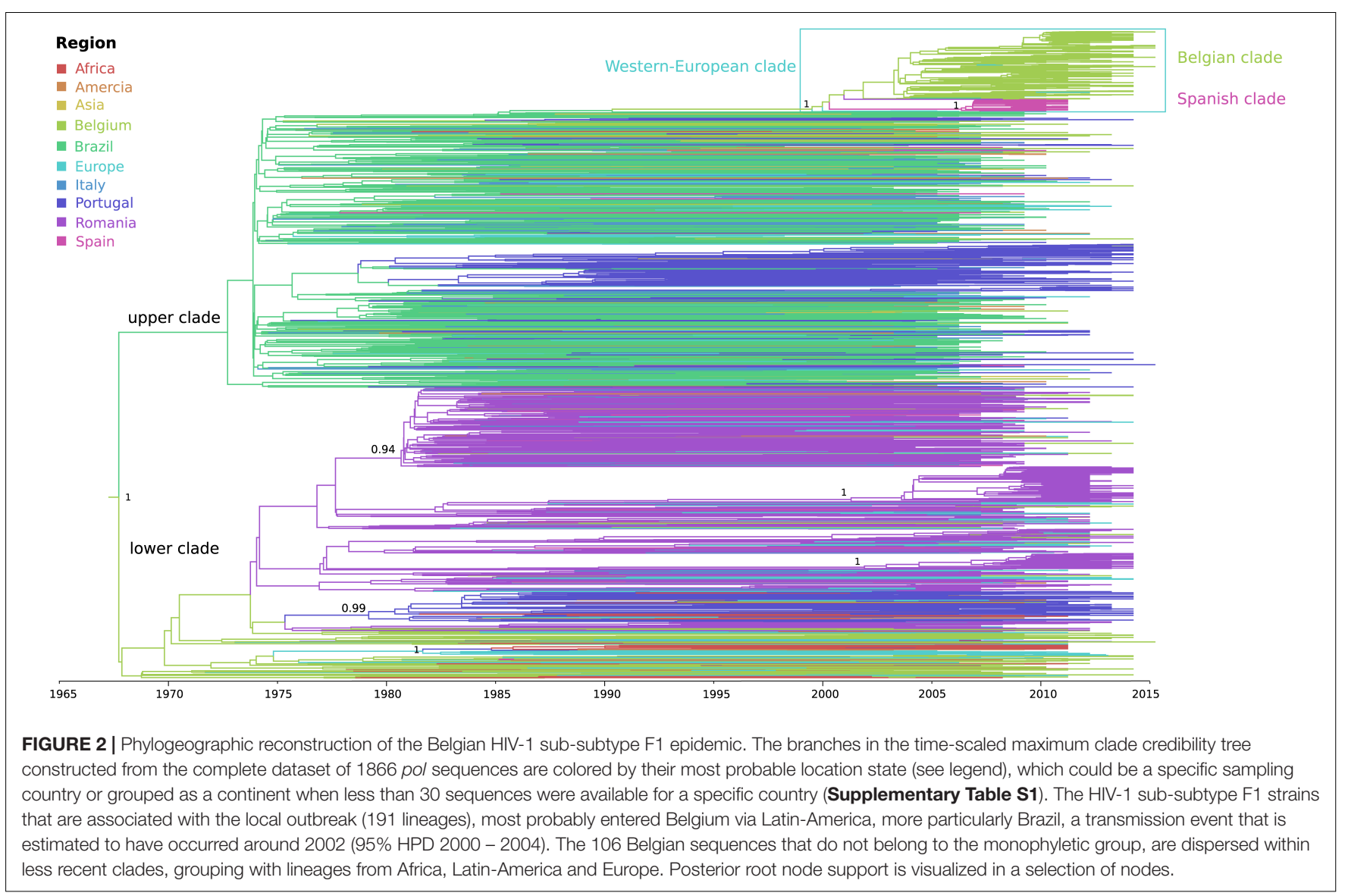

dispersed across the tree and are intermixed among viral lineages from Africa, Latin-America and Europe (Figures 2, 3). Several other strains from Sub-Saharan migrants residing in Belgium but who originated from the Democratic Republic of Congo (DRC), Congo, and Angola occupied the most basal positions of the tree (Figure 2 lower clade, Figure 3). They cluster with African lineages that probably migrated to Europe between 1972 and 1977 (95\% HPD 1969-1980) and that were at the origin of several larger sub-subtype F1 outbreaks in Europe, more specifically in Portugal (1979, 95\% HPD 1976-1982), Romania (1981, 95\% HPD 1978-1983; 2001, 95\% HPD 1996-2006; and 2002, 95\% HPD 1998-2006), and Bulgaria (1982, HPD 1979-1985). As, we defined a location trait according to country of sampling in our analysis, the over-representation of Belgian sequences - probably to a large extent reflecting African diversity - led Belgium to be inappropriately assigned as the ancestor location of this lower clade that contained many African, Romanian, Portuguese, and other European lineages (Figure 2 lower clade).

\section{Socio-Epidemiological Correlates With the Sub-Subtype F1 Outbreak in Belgium}

As one or only a few sub-subtype F1 lineages have successfully established local transmissions among 191 of 297 people diagnosed with a sub-subtype F1 infection in Belgium (64\%), we used univariate and multivariate logistic regression to identify socio-epidemiological variables that were associated with this successful sub-subtype F1 outbreak in Belgium (Table 1). HIV1 patients who were diagnosed with a sub-subtype F1 infection at the AIDS reference laboratories in Antwerp (OR 6.80, 95\% CI 3.86-11.99), Leuven (OR 4.22, 95\% CI 1.59-11.16) or more broadly in the region of Flanders (OR 7.28, 95\% CI 4.23-12.53) had significantly higher odds of belonging to this F1 outbreak, compared to people who were diagnosed with a sub-subtype F1 infection elsewhere in Belgium. In addition, many other variables of the sub-subtype F1 infections diagnosed in Belgium displayed significantly higher odds of being part of the outbreak, of which the most informative were male gender (OR 52.42, 95\% CI 19.90-138.08), infection in MSM (combining homosexuals and bisexuals) (OR 34.8, 95\% CI 16.0-75.6), probable infection in Belgium (OR 26.67, 95\% CI 9.59-74.15), born in Belgium (OR $12.10,95 \%$ CI 6.35-23.08), recent infection stage at diagnosis (OR 7.19, 95\% CI 2.88-17.95) and higher viral load (OR 2.36, 95\% CI 1.78-3.13). In multivariate analysis, sub-subtype F1 infections in MSM and in people born in Belgium remained to display a higher likelihood for belonging to the sub-subtype F1 Belgian outbreak [OR 7.82, 95\% CI 3.80-16.11 and OR 2.01 (1.06-3.78), respectively]. The variables sampling region, ARL in Belgium, gender, country of birth and sexual risk group were visualized for all Belgian sequences in a circular representation of the phylogenetic tree (Figure 3). 

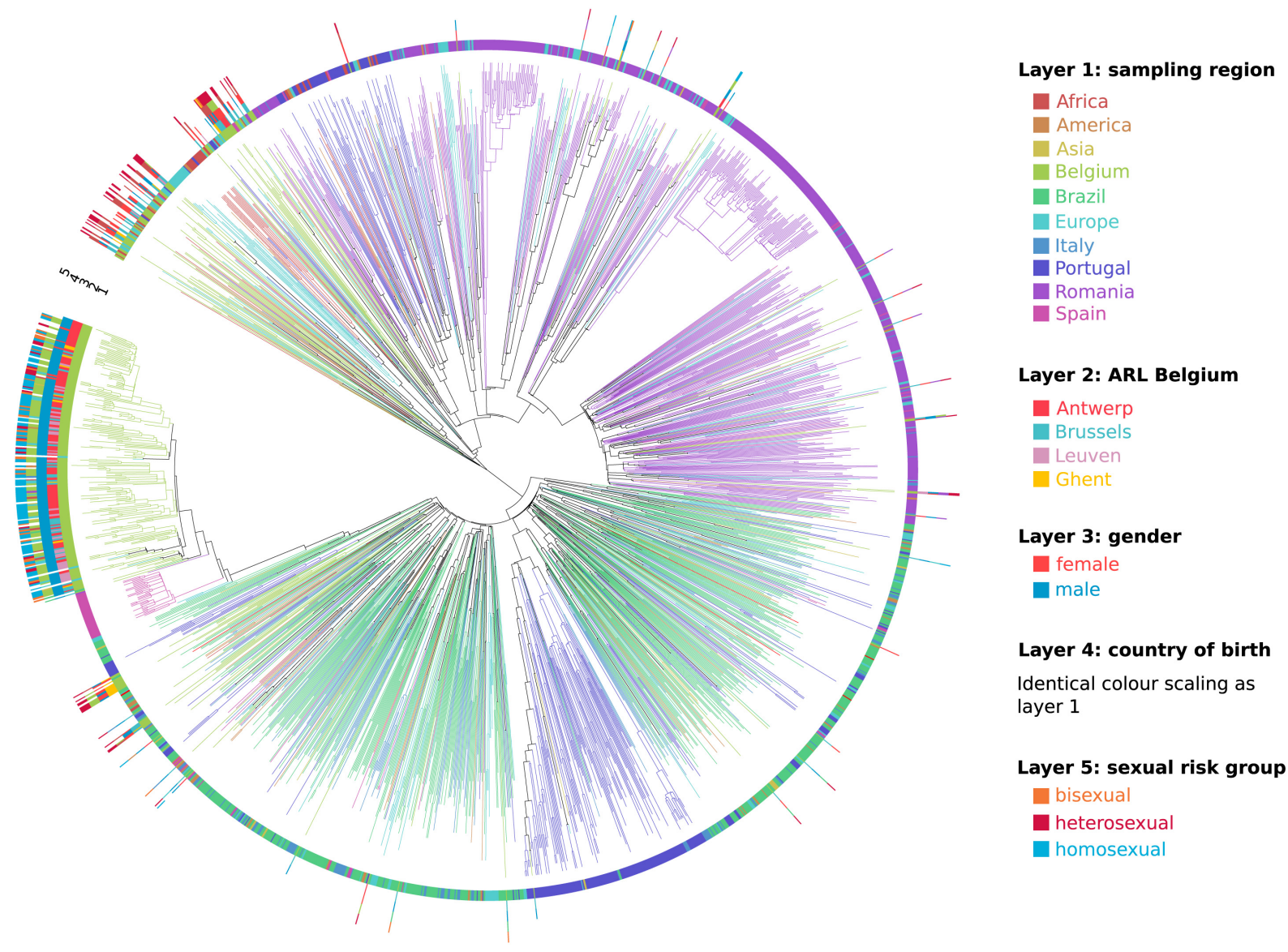

Layer 2: ARL Belgium

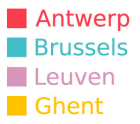

Layer 3: gender

female

$\square$ male

Layer 4: country of birth

Identical colour scaling as layer 1

\section{Layer 5: sexual risk group bisexual \\ neterosexual \\ nomosexual}

FIGURE 3 | Circular representation of the maximum clade credibility tree of the Belgian sub-subtype F1 epidemic within a global context. Branches in the tree are colored according to sampling region (identical coloring scaling as in Figure 2), which is also displayed in layer 1. For the HIV-1 sub-subtype F1 strains that were sampled at the AIDS reference laboratories (ARL) in Belgium, the location of testing was displayed in layer 2, gender in layer 3, country of birth in layer 4, and sexual risk group in layer 5 . This detailed information was largely lacking for the sequences sampled outside Belgium.

\section{Reduced Onward Transmission of Sub-Subtype F1 Strains Coincident With an Earlier Initiation of Antiretroviral \\ Therapy}

Since the onset of the sub-subtype F1 outbreak in Belgium (2002, 95\% HPD 2000-2004), we covered a time period where antiretroviral therapy was initially still deferred in asymptomatic patients with CD4 count above 350 cells $/ \mathrm{mm}^{3}$ (European AIDS Clinical Society, 2005). This general practice gradually switched to the consideration of antiretroviral therapy for asymptomatic patients with CD4 counts between 350 and 500 cells $/ \mathrm{mm}^{3}$ (European AIDS Clinical Society, 2007) and above 500 cells $/ \mathrm{mm}^{3}$ (European AIDS Clinical Society, 2013). Given the benefits of antiretroviral therapy in reducing infectivity, we wanted to quantify transmission dynamics through time using a birthdeath model and monitor the trends in therapy uptake in our specific setting. Therapy and diagnosis information was available for 147 HIV-1 patients whose viral strains belonged to the sub-subtype F1 outbreak in Belgium (77\%, 147/191). The delay in therapy initiation decreased from a median of 1011 days (IQR 368-1683) or almost 3 years for patients diagnosed in 2005 and 2006 to a median of 61 days (IQR 26208) or about 2 months for patients diagnosed in 2013 and 2014 (Figure 4). In the birth-death model, assuming a constant $65 \%$ sampling proportion, the number of expected secondary infections per infected person (effective reproductive number $R_{e}$ ) was estimated to be a median of 3 in 2005 and gradually declined to a median of $1.5 \mathrm{in} 2011$, after which a substantial drop to below 1 was observed (Figure 5). During this time window, the median infectious time also declined and the number of expected secondary infections per infected person and per year (transmission rate) decreased from a median of 1 to well below 1. Sensitivity analysis using a constant sampling proportion of $80 \%$, as the diagnosis and sequencing rate is expected to be higher in MSM, showed similar results and suggests that the estimates are relatively robust to prior specification on the sampling proportion.

\section{DISCUSSION}

Human immunodeficiency virus type 1 subtype $F$ most likely emerged from the early 20th century HIV-1 diversity 
TABLE 1 | Characteristics of people diagnosed with an HIV-1 sub-subtype F1 infection in Belgium and factors associated with this local outbreak.

\begin{tabular}{|c|c|c|c|c|c|c|}
\hline \multirow[t]{2}{*}{ Characteristic } & \multirow[t]{2}{*}{$\begin{array}{l}\text { Local outbreak } \\
\qquad(n=191)\end{array}$} & \multirow[t]{2}{*}{ Other $(n=106)$} & \multicolumn{2}{|l|}{$\begin{array}{l}\text { Univariate logistic } \\
\text { regression }\end{array}$} & \multicolumn{2}{|c|}{$\begin{array}{l}\text { Multivariate logistic } \\
\text { regression }\end{array}$} \\
\hline & & & OR (95\% Cl) & $p$ & OR $(95 \% \mathrm{Cl})$ & $p$ \\
\hline \multicolumn{7}{|c|}{ AIDS Reference Laboratory [\% (n)] } \\
\hline Antwerp & $61.3(117)$ & $18.9(20)$ & $6.80(3.86-11.99)$ & $<0.0001$ & & \\
\hline Brussels & $16.7(32)$ & $59.4(63)$ & $0.14(0.08-0.24)$ & $<0.0001$ & & \\
\hline Leuven & $17.3(33)$ & $4.7(5)$ & $4.22(1.59-11.16)$ & 0.0037 & & \\
\hline Ghent & $4.7(9)$ & $17.0(18)$ & $0.24(0.10-0.56)$ & 0.0009 & & \\
\hline \multicolumn{7}{|l|}{ Region [\% (n)] } \\
\hline Flanders & $83.3(159)$ & $40.6(43)$ & $7.28(4.23-12.53)$ & $<0.0001$ & & \\
\hline \multicolumn{7}{|l|}{ Gender [\% (n)] } \\
\hline Male & $97.4(186)$ & $41.5(44)$ & $52.42(19.90-138.08)$ & $<0.0001$ & & \\
\hline \multicolumn{7}{|l|}{ Age at diagnosis [years] } \\
\hline Median (IQR) & $34[29,41](190)$ & $34[27,40](92)$ & $1.03(1.00-1.06)$ & 0.0343 & & \\
\hline \multicolumn{7}{|l|}{ Diagnosis date [dd-mm-yyyy] } \\
\hline \multirow[t]{2}{*}{ Median [IQR] (n) } & 09-05-2012 & $24-1-2008$ & $1.33(1.23-1.45)$ & $<0.0001$ & & \\
\hline & $\begin{array}{c}{[21-03-2010,} \\
23-11-2013](190)\end{array}$ & $\begin{array}{c}{[3-4-2002,} \\
31-10-2011](92)\end{array}$ & & & & \\
\hline \multicolumn{7}{|c|}{ Infection stage at diagnosis [\% (n)] } \\
\hline Acute & $10.1(16 / 158)$ & $2.2(1 / 45)$ & $4.96(0.64-38.45)$ & 0.1256 & & \\
\hline Recent & $52.5(83 / 158)$ & $13.3(6 / 45)$ & $7.19(2.88-17.95)$ & $<0.0001$ & & \\
\hline Chronic & $37.4(59 / 158)$ & $84.5(38 / 45)$ & $0.11(0.05-0.26)$ & $<0.0001$ & & \\
\hline Unknown & $17.2(33)$ & $57.5(61)$ & & & & \\
\hline \multicolumn{7}{|l|}{ Country of birth [\% (n)] } \\
\hline Belgium & $77.8(123 / 158)$ & $22.5(18 / 80)$ & $12.10(6.35-23.08)$ & $<0.0001$ & $2.01(1.06-$ & 0.0316 \\
\hline Other & $22.2(35 / 158)$ & $77.5(62 / 80)$ & & & $3.78)$ & \\
\hline Unknown & $17.2(33)$ & $24.5(26)$ & & & & \\
\hline \multicolumn{7}{|c|}{ Probable country of infection $[\%(n)]$} \\
\hline Belgium & $95.2(120 / 126)$ & $42.9(18 / 42)$ & $26.67(9.59-74.15)$ & $<0.0001$ & & \\
\hline Other & $4.8(6 / 126)$ & $57.1(24 / 42)$ & & & & \\
\hline Unknown & $34.0(65)$ & $60.4(64)$ & & & & \\
\hline \multicolumn{7}{|l|}{ Sexual risk group [\% (n)] } \\
\hline Homosexual contacts & $69.2(110 / 159)$ & $9.7(7 / 72)$ & 20.85 (8.92-48.73) & $<0.0001$ & & $<0.0001$ \\
\hline Bisexual contacts & $18.2(29 / 159)$ & $7.0(5 / 72)$ & $2.99(1.11-8.08)$ & & & \\
\hline Heterosexual contacts & $12.6(20 / 159)$ & $83.3(60 / 72)$ & $0.03(0.01-0.06)$ & 0.0308 & & \\
\hline Men-having-sex-with-men & $87.4(139 / 159)$ & $16.7(12 / 72)$ & $34.8(16.0-75.6)$ & $<0.0001$ & $7.82(3.80-$ & \\
\hline Unknown & $16.8(32)$ & $32.1(34)$ & & $<0.0001$ & 16.11) & \\
\hline \multicolumn{7}{|l|}{ Viral load at sequencing $\left[\log _{10}\right.$} \\
\hline copies/ml] Median [IQR] (n) & 5.18 [4.61-5.79] (191) & $4.36[3.75,4.92](101)$ & $2.36(1.78-3.13)$ & $<0.0001$ & & \\
\hline \multicolumn{7}{|l|}{ CD4+ at sequencing [cells/ $\mu l]$} \\
\hline Median [IQR] (n) & 488 [358-645] (184) & $334[175,528](89)$ & $1.00(1.00-1.00)$ & $<0.0001$ & & \\
\hline \multicolumn{7}{|l|}{ CD8+ at sequencing [cells/ $\mu l]$} \\
\hline Median [IQR] $(n)$ & 1059 [697-1598] (173) & $1041[778,1390](67)$ & $1.00(1.00-1.00)$ & 0.2980 & & \\
\hline
\end{tabular}

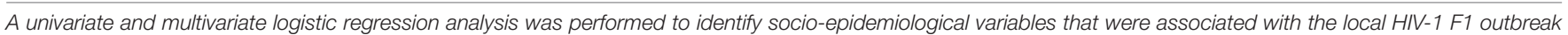
in Belgium ( $n=191)$. A p-value of < 0.05 was considered to be significant. Abbreviations: $n$, number, IQR, interquartile range, OR, odds ratio, Cl, confidence interval.

in the DRC, where its current prevalence remains less than 5\% (Vidal et al., 2000; Faria et al., 2014; Rodgers et al., 2017). This subtype accounts for less than $1 \%$ of HIV-1 infections worldwide and is subdivided into two sub-subtypes $\mathrm{F} 1$ and F2, of which the detection of the latter remains mostly restricted to Cameroon (Brennan et al., 2008; Hemelaar et al., 2011). In contrast, sub-subtype F1 strains have spread to other Central
African countries, reaching a particularly high prevalence of $14 \%$ in Angola (Afonso et al., 2012). In Belgium, the burden of sub-subtype F1 infections among newly diagnosed HIV-1 patients increased to $11.1 \%$ in 2015. The aim of this study was to characterize the epidemic dynamics of this non-B subtype epidemic in Belgium by using phylogenetic approaches and the analysis of socio-epidemiological variables. 


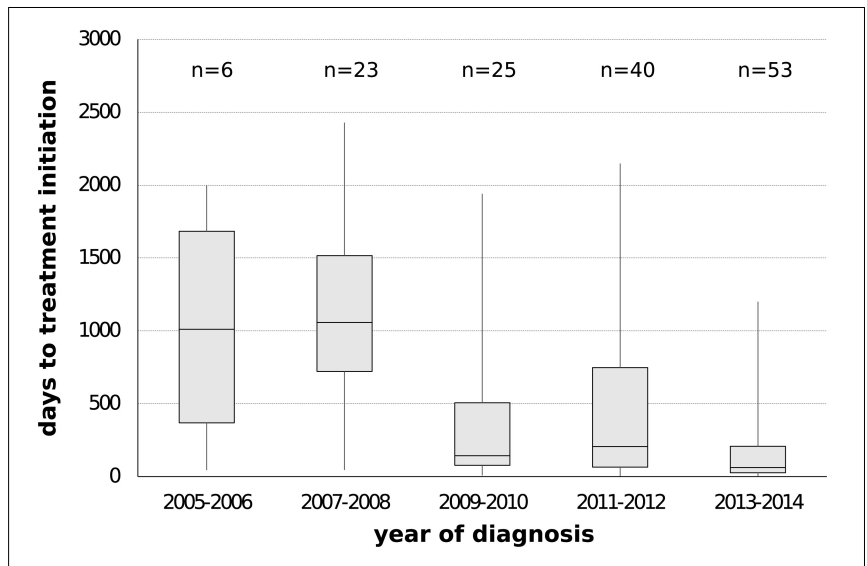

FIGURE 4 | Delay in antiretroviral treatment among people infected with sub-subtype F1 strains belonging to the local outbreak in Belgium. The number of days between diagnosis date and treatment initiation date were plotted according to 2-year intervals of diagnosis date. Information on prescribed antiviral therapy and diagnosis date was available for $77 \%$ of the HIV-1 patients belonging to the local outbreak in Belgium (147/191).

\section{The Dissemination of HIV-1 Sub-Subtype F1 Across the World}

Our and other phylogenetic analyses confirmed that the most basal F1 sequences were isolated from people originating from Central African countries and that the major migration events out of Africa occurred in the 1970s and early 1980s seeding some larger F1 epidemics over the world (Mehta et al., 2011; Bello et al., 2012; Lai et al., 2014). During the Angolan civil war that started in 1975 and lasted for more than 16 years, an extensive migration of troops, settlers and refugees was observed between Angola, DRC, Portugal, Brazil, Cuba, Russia, and several Eastern-European countries and this is considered as an important factor in the global dissemination of F1. In Brazil, sub-subtype F1 infections have reached prevalence rates of more than $7 \%$ and the strains are currently circulating among various risk groups (Brindeiro et al., 2003). It is the most probable epicenter of F1-related epidemics within the Southern Cone and one of the sources of the Portuguese sub-subtype F1 epidemic (Aulicino et al., 2007; Bello et al., 2007, 2012; Carvalho et al., 2015). Unrelated to the Brazilian epidemic, sub-subtype F1 was initially introduced and spread among adult heterosexuals in Romania. In this Eastern-European country, it subsequently reached a very high prevalence rate of more than $70 \%$ due to several major outbreaks among children in orphanages and people using intravenous drugs (Dumitrescu et al., 1994; Bandea et al., 1995; Apetrei et al., 1998; Op De Coul et al., 2000; Guimarães et al., 2009; Paraschiv et al., 2009; Mehta et al., 2011; Mbisa et al., 2012; Niculescu et al., 2014). Both Brazil and Romania were at the origin of the F1 epidemic in Italy, where Brazilian strains contributed to many more introduction events characterized with a more extensive spread (Lai et al., 2014). Since then, the prevalence of sub-subtype F1 increased gradually among Italian descendants and mainly among self-declared heterosexual men, although phylogenetic analyses suggested that some of these self-declared heterosexual men might have been infected through homosexual contact (Bracciale et al., 2009; Lai et al., 2012, 2014).

Although our and a previous study indicated that another sub-subtype F1 lineage migrated from Brazil to Europe during the 1990s, this lineage obtained only more recently epidemic proportions in two countries, respectively, about 16 years ago in Belgium and 12 years ago in Spain (Paraskevis et al., 2015). In both instances, F1 was introduced in local MSM networks, a factor probably important in its subsequent spread and circulation at high rates (Thomson et al., 2012). Our analyses did not indicate any intermixing between both epidemics and only sporadic and putative exports from Belgium to Luxembourg (one) and Germany (two) were detected. This is in agreement with another study that traced the HIV-1 subtype B mobility in Europe and that characterized Belgium primarily as a sink of HIV-1 lineages rather than a source (Paraskevis et al., 2009). Although, we confirmed previous findings on the global spread of HIV-1 sub-subtype F1, our sampling approach might not have been sensitive enough to capture the specific migration events that resulted into these two large outbreaks or into extensive onward transmission to other countries. For our analyses, we collected HIV-1 pol sequences from countries with a known high burden of F1 infections or a potential role in the global spread of sub-subtype F1. In this dataset, Belgium, Romania, Portugal, Luxembourg, and Bulgaria were the best-represented countries as they contributed a dense sampling, whereas Italy provided a down-sampling proportional to their local subsubtype F1 diversity. However, other countries might have been undersampled as they were represented by sequences that were received from single cohorts or by sequences that were collected from public databases. Such sample biases call for caution in interpreting the results of phylogeographic estimates.

\section{The Belgian HIV-1 Sub-Subtype F1 Outbreak}

The first HIV-1 sub-subtype F1 infections within the local outbreak cluster were diagnosed in 2005. It concerned one chronic infection in Leuven and one infection with unknown status in Antwerp, suggesting a time of about 3 years between the estimated introduction in Belgium (2002, 95\% HPD 20002004) and the first case reported by the Belgian ARL. Although there is universal access to HIV-1 health care in Belgium and we included all patients for whom genotypic drug resistance testing indicated a sub-subtype F1 infection, whether at baseline or at therapeutic failure, our sequencing efforts in routine practice only cover approximately $60 \%$ of all newly HIV-1 diagnoses registered in the national database. However, we believe that we do capture the majority of patients who are contributing to the local spread, as sub-subtype F1 is mainly circulating among MSM, a group who is well engaged in medical care (Van Beckhoven et al., 2015). Any HIV-1 patient who seeks medical care in Belgium will receive a confirmation of his/her infection and will therefore be registered in the national database. Therefore, we believe that a large proportion of the people living with HIV-1 in Belgium who do not receive a baseline resistance test at diagnosis are people with an infection that was already 

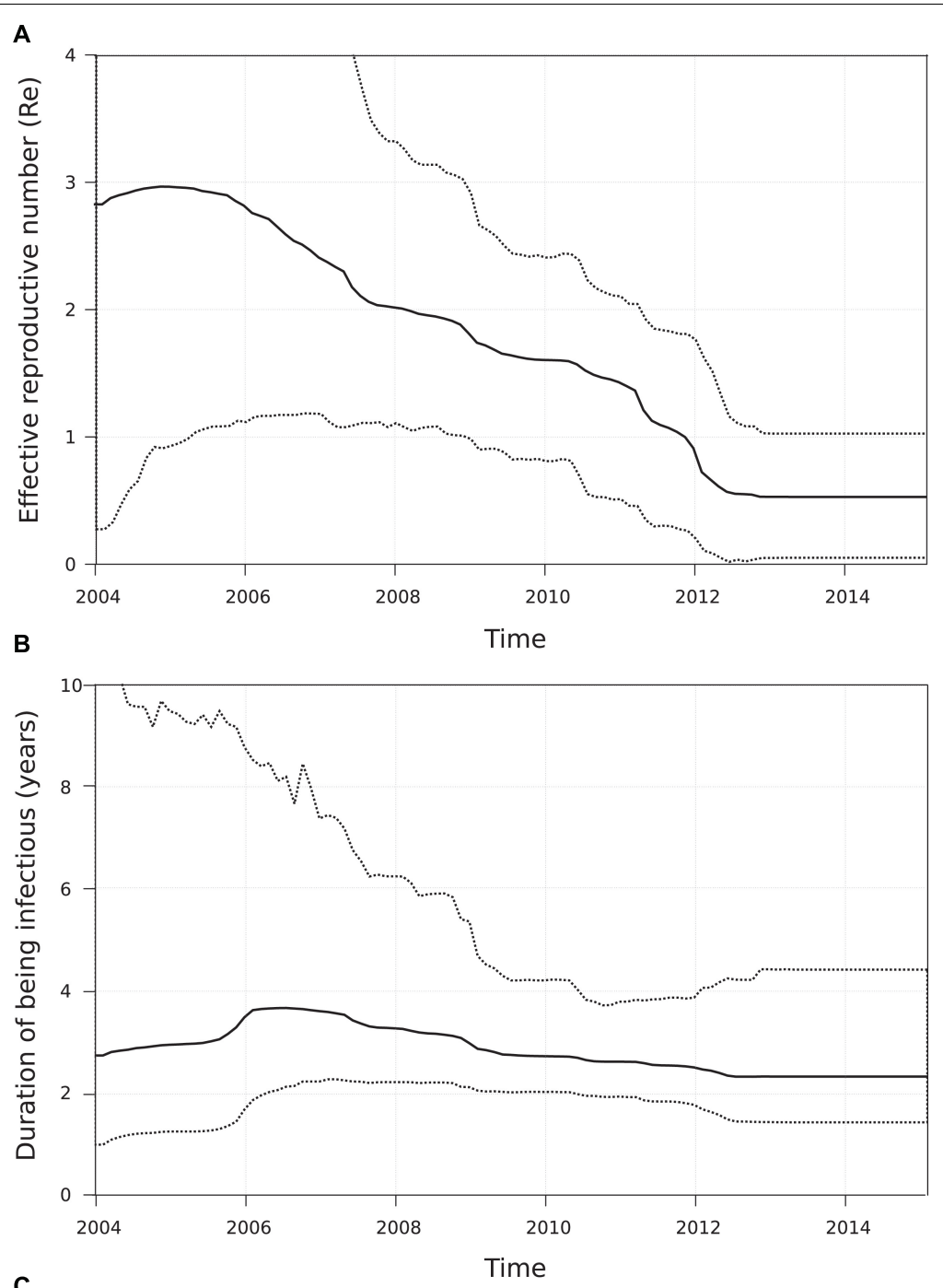

C

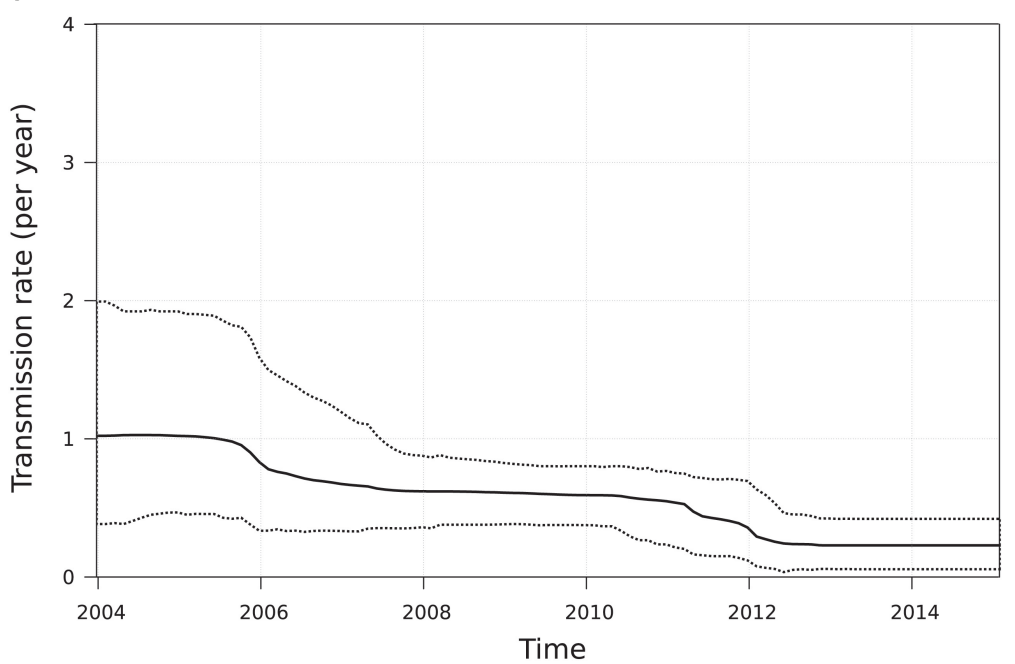

FIGURE 5 | Birth-death model applied to the sub-subtype F1 outbreak in Belgium. For all patients belonging to the large Belgian cluster ( $n=191$ ), the number of secondary infections per infected person (effective reproductive number Re, panel (A)), the median infectious time in years (panel (B)) and the number of secondary infections per infected person per year (transmission rate, panel (C)) was estimated. After the onset of the outbreak both Re and transmission rate started to decline, with the largest drop occurring after 2011. 
acquired and diagnosed abroad and who are already on successful antiretroviral treatment upon arrival in Belgium, or these people are only temporarily in the country and do not enter the HIV-1 health care system in Belgium.

With the dense sampling of our local epidemic at hand, we aimed to estimate the effective reproductive number $R_{e}$, the transmission (birth) rates and the time of being infectious in our viral population using birth-death modeling in order to gain insights into the dynamics of the outbreak. The initial high $R_{\mathrm{e}}$-value, high transmission rate and long times of being infectious were in agreement with the explosive increase of sub-subtype F1 infections in our national cohort. However, the mean $R_{\mathrm{e}}$-value declined substantially from 2012 onward and this event was associated with lower transmission rates and shorter durations of being infectious. These observations may be explained by the policy changes around that time when therapy eligibility thresholds for asymptomatic HIV-1 patients became less strict in Belgium. Indeed, medical records showed a remarkable dent in the median treatment delay among people belonging to our outbreak around 20092010. Although this relationship does not demonstrate causality, it is supported by studies where viral suppression by antiretroviral therapy has shown to be effective in preventing HIV-1 transmission among serodiscordant heterosexual and MSM couples (Cohen et al., 2011; Rodger et al., 2016; Bavinton et al., 2018). However, the relevant changes in transmission dynamics were not yet reflected in the number of new sub-subtype F1 diagnoses up to the start of 2015. This could suggest that the effects were too recent to be fully captured by our surveillance data of new diagnoses or that the conditions were not yet fully optimal to achieve the full potential of treatment as prevention in this outbreak (Okano et al., 2016). Although a substantial proportion of our outbreak was diagnosed at an acute and recent stage of infection, we should aim to increase testing and diagnosis rates among people at risk of acquiring HIV-1 and treat them without any delay. In this respect, the INSIGHT START study resolved the last concerns for immediate initiation of antiretroviral therapy in asymptomatic HIV-1 infections as this study showed that this strategy was superior to deferral until the CD4 count declined to 350 cells $/ \mathrm{mm}^{3}$ and that it was not associated with an increased rate of adverse events (INSIGHT START Study Group et al., 2015).

In conclusion, this study revealed that sub-subtype F1 strains were introduced in Belgium around 2002 and that during a decade they spread exponentially among MSM who were mainly from Belgian descent, residing in Flanders and diagnosed at a recent stage of infection. Our birth-death phylodynamic estimates are in line with the expectations of the effectiveness of treatment as prevention because we identify a decline in the onward transmission of sub-subtype F1 strains that coincides with the implementation of earlier initiation of antiretroviral therapy in our cohort. Further follow-up is planned, especially within the context of the roll-out of antiretroviral therapy in early asymptomatic HIV-1 infections and of the implementation of pre-exposure prophylaxis which is reimbursed to people who are at increased risk for HIV-1 infection since June 2017.

\section{DATA AVAILABILITY}

The datasets generated for this study can be found in GenBank, MK458435-MK458523.

\section{AUTHOR CONTRIBUTIONS}

KVL conceived and designed the study. LV, KF, IA, CB, LD, CS-D, SGR, PG, FI, RK, JR, MS, SP, RP, MP, AS, EV, SVW, MVR, $\mathrm{CV}, \mathrm{A}-\mathrm{MV}$, and KVL collected and analyzed the epidemiological and viral data. LV, LC, TS, and PL performed the phylogenetic analyses and inferences. LV, LC, and KVL wrote the first draft of the manuscript. All authors contributed to manuscript revision, read and approved the submitted version.

\section{FUNDING}

This work was supported in part by grants from the Fonds voor Wetenschappelijk Onderzoek Vlaanderen (FWO) (G.0692.14N, G.0E84.16N, G.0B23.17N) and KU Leuven (Program Financing No. PF/10/018), and by the AIDS Reference Laboratories that receive support from the Belgian Ministry of Social Affairs through a fund within the Health Insurance System. The computational resources and services used in this work were partly provided by the Hercules Foundation and the Flemish Government department EWI-FWO Krediet aan Navorsers (KAN2012 1.5.249.12). PL acknowledges funding from the European Research Council under the European Union's Horizon 2020 Research and Innovation Programme (grant agreement no. 725422-ReservoirDOCS), from the Special Research Fund, KU Leuven ("Bijzonder Onderzoeksfonds," KU Leuven, OT/14/115), and the Research Foundation - Flanders ("Fonds voor Wetenschappelijk Onderzoek - Vlaanderen," G.0662.15N, G.0D51.17N, and G.0B93.17N). TS was supported in part by the European Research Council under the 7th Framework Program of the European Commission (PhyPD, Grant Agreement 335529).

\section{ACKNOWLEDGMENTS}

This study was the result of a collaborative effort. We would like to thank Andrea-Clemencia Pineda-Peña, Yoeri Schrooten, Fossie Ferreira, and Tim Dierickx for their technical assistance, and the patients and clinicians at the AIDS Reference Centers who contributed data. We would like to acknowledge BREACH that facilitates HIV research among different actors in Belgium.

\section{SUPPLEMENTARY MATERIAL}

The Supplementary Material for this article can be found online at: https://www.frontiersin.org/articles/10.3389/fmicb.2019. 00613/full\#supplementary-material 


\section{REFERENCES}

Abecasis, A. B., Wensing, A. M., Paraskevis, D., Vercauteren, J., Theys, K., Van de Vijver, D. A., et al. (2013). HIV-1 subtype distribution and its demographic determinants in newly diagnosed patients in Europe suggest highly compartmentalized epidemics. Retrovirology 10:7. doi: 10.1186/17424690-10-7

Afonso, M. J., Bello, G., Guimarães, M. L., Sojka, M., and Morgado, M. G. (2012). HIV-1 diversity and transmitted drug resistance mutations among patients from the North, Central and South Regions of Angola. PLoS One 7:e42996. doi: 10.1371/journal.pone.0042996

Alexiev, I., Shankar, A., Wensing, A. M., Beshkov, D., Elenkov, I., Stoycheva, M., et al. (2015). Low HIV-1 transmitted drug resistance in Bulgaria against a background of high clade diversity. J. Antimicrob. Chemother. 70, 1874-1880. doi: 10.1093/jac/dkv011

Apetrei, C., Necula, A., Holm-Hansen, C., Loussert-Ajaka, I., Pandrea, I., Cozmei, C., et al. (1998). HIV-1 diversity in Romania. AIDS 12, 1079-1085. doi: 10.1097/00002030-199809000-00016

Aulicino, P. C., Bello, G., Rocco, C., Romero, H., Mangano, A., Morgado, M. G., et al. (2007). Description of the first full-length HIV type 1 subtype F1 strain in Argentina: implications for the origin and dispersion of this subtype in South America. AIDS Res. Hum. Retroviruses 23, 1176-1182. doi: 10.1089/aid.2007. 0038

Ayres, D. L., Darling, A., Swickl, D. J., Beerli, P., Holder, M. T., Lewis, P. O., et al. (2012). BEAGLE : an application programming interface and high-performance computing library for statistical phylogenetics. Syst. Biol. 61, 170-173. doi: $10.1093 /$ sysbio/syr 100

Bandea, C. I., Ramos, A., Pieniazek, D., Pascu, R., Tanuri, A., G, et al. (1995). Epidemiologic and evolutionary relationships between Romanian and Brazilian HIV-subtype F strains. Emerg. Infect. Dis. 1, 91-93. doi: 10.3201/eid0103.950305

Bavinton, B. R., Pinto, A. N., Phanuphak, N., Grinsztejn, B., Prestage, G. P., Zablotska-Manos, I. B., et al. (2018). Viral suppression and HIV transmission in serodiscordant male couples: an international, prospective, observational, cohort study. Lancet HIV 5, e438-e447. doi: 10.1016/S2352-3018(18)30132-2

Bello, G., Afonso, J. M., and Morgado, M. G. (2012). Phylodynamics of HIV-1 subtype F1 in Angola, Brazil and Romania. Infect. Genet. Evol. 12, 1079-1086. doi: 10.1016/j.meegid.2012.03.014

Bello, G., Eyer-Silva, W. A., Couto-Fernandez, J. C., Guimarães, M. L., ChequerFernandez, S. L., Teixiera, S. L., et al. (2007). Demographic history of HIV-1 subtypes B and F in Brazil. Infect. Genet. Evol. 7, 263-270. doi: 10.1016/j.meegid. 2006.11.002

Bennett, D., Camacho, R. J., Otelea, D., Kuritzkes, D. R., Fleury, H., Kiuchi, M. et al. (2009). Drug resistance mutations for surveillance of transmitted HIV1 drug-resistance: 2009 update. PLoS One 4:e4724. doi: 10.1371/journal.pone. 0004724

Bouckaert, R., Heled, J., Kühnert, D., Vaughan, T., Wu, C. H., Xie, D., et al. (2014). BEAST 2: a software platform for Bayesian evolutionary analysis. PLoS Comput. Biol. 10:e1003537. doi: 10.1371/journal.pcbi.1003537

Bracciale, L., Colafigli, M., Zazzi, M., Corsi, P., Meraviglia, P., Micheli, V., et al. (2009). Prevalence of transmitted HIV-1 drug resistance in HIV-1 infected patients in Italy: evolution over 12 years and predictors. J. Antimicrob. Chem 64, 607-615. doi: 10.1093/jac/dkp246

Brennan, C. A., Bodelle, P., Coffey, R., Devare, S. G., Golden, A., Hackett, J. Jr., et al. (2008). The prevalence of diverse HIV-1 strains was stable in Cameroonian blood donors from 1996 to 2004. J. Acquir. Immune Defic. Syndr. 49, 432-439. doi: 10.1097/QAI.0b013e31818a6561

Brindeiro, R. M., Diaz, R. S., Sabino, E. C., Morgado, M. G., Pires, I. L., Brigido, L., et al. (2003). Brazilian network for HIV drug resistance surveillance (HIVBResNet): a survey of chronically infected individuals. AIDS 17, 1063-1069. doi: 10.1097/00002030-200305020-00016

Carvalho, A., Costa, P., Triunfante, V., Branca, F., Rodrigues, F., Santos, C. L., et al. (2015). Analysis of a local HIV-1 epidemic in Portugal highlights established transmission of non-B and non-G subtypes. J. Clin. Microbiol. 53, 1506-1514 doi: 10.1128/JCM.03611-14

Chalmet, K., Staelens, D., Blot, S., Dinakis, S., Pelgrom, J., Plum, J., et al. (2010). Epidemiological study of phylogenetic transmission clusters in a local HIV-1 epidemic reveals distinct differences between subtype B and non-B infections. BMC Infect. Dis. 10:262. doi: 10.1186/1471-2334-10-262
Cid-Silva, P., Margusino-Framiñán, L., Balboa-Barreiro, V., Martín-Herranz, I., Castro-Iglesias, Á, Pernas-Souto, B., et al. (2018). Initial treatment response among HIV subtype $\mathrm{F}$ infected patients who started antiretroviral therapy based on integrase inhibitors. AIDS 32, 121-125. doi: 10.1097/QAD. 0000000000001679

Cohen, M. S., Chen, Y. Q., McCauley, M., Gamble, T., Cohen, M. S., Chen, Y. Q., et al. (2011). Prevention of HIV-1 infection with early antiretroviral therapy. N. Engl. J. Med. 365, 493-505. doi: 10.1056/NEJMoa1105243

Dumitrescu, O., Kalish, M. L., Kliks, S. C., Bandea, C. I., and Levy, J. A. (1994). Characterization of human immunodeficiency virus type 1 isolates from children in Romania: identification of a new envelope subtype. J. Infect. Dis. 169, 281-288. doi: 10.1093/infdis/169.2.281

Edgar, R. C. (2004). MUSCLE: multiple sequence alignment with high accuracy and high throughput. Nucleic Acids Res. 32, 1792-1797. doi: 10.1093/nar/ gkh340

European AIDS Clinical Society (2005). EACS Guidelines. [Internet]. Available at: http://www.eacsociety.org/guidelines/eacs-guidelines/eacs-guidelines.html

European AIDS Clinical Society (2007). EACS Guidelines. [Internet]. Available at: http://www.eacsociety.org/guidelines/eacs-guidelines/eacs-guidelines.html

European AIDS Clinical Society (2013). EACS Guidelines. [Internet]. Available at: http://www.eacsociety.org/guidelines/eacs-guidelines/eacs-guidelines.html

Faria, N. R., Rambaut, A., Suchard, M. A., Baele, G., Bedford, T., Ward, M. J., et al. (2014). HIV epidemiology. The early spread and epidemic ignition of HIV-1 in human populations. Science 346, 56-61. doi: 10.1126/science.1256739

Fransen, K., Buvé, A., Nkengasong, J. N., Laga, M., and van der Groen, G. (1996). Longstanding presence in Belgians of multiple non-B HIV-1 subtypes. Lancet 347:1403. doi: 10.1016/S0140-6736(96)91042-9

Frentz, D., Boucher, C. A., Assel, M., De Luca, A., Fabbiani, M., Incardona, F., et al. (2010). Comparison of HIV-1 genotypic resistance test interpretation systems in predicting virological outcomes over time. PLoS One 5:e11505. doi: 10.1371/journal.pone.0011505

Guimarães, M. L., Vicente, A. C., Otsuki, K., da Silva, R. F., Francisco, M., da Silva, F. G., et al. (2009). Close phylogenetic relationship between Angolan and Romanian HIV-1 subtype F1 isolates. Retrovirology 6:39. doi: 10.1186/17424690-6-39

Hemelaar, J., Gouws, E., Ghys, P. D., Osmanov, S., and WHO-UNAIDS Network for HIV Isolation, and Characterisation (2011). Global trends in molecular epidemiology of HIV-1 during 2000-2007. AIDS 25, 679-689. doi: 10.1097/ QAD.0b013e328342ff93

INSIGHT START Study Group, Lundgren, J. D., Babiker, A. G., Gordin, F., Emery, S., Grund, B., et al. (2015). Initiation of antiretroviral therapy in early asymptomatic HIV infection. N. Engl. J. Med. 373, 795-807. doi: 10.1056/ NEJMoa1506816

Ivanov, I. A., Beshkov, D., Shankar, A., Hanson, D. L., Paraskevis, D., Georgieva, V., et al. (2013). Detailed molecular epidemiologic characterization of HIV-1 infection in Bulgaria reveals broad diversity and evolving phylodynamics. PLoS One 8:e59666. doi: 10.1371/journal.pone.0059666

Junqueira, D. M., and Almeida, S. E. (2016). HIV-1 subtype B: traces of a pandemic. Virology 495, 173-184. doi: 10.1016/j.virol.2016.05.003

Lai, A., Ciccozzi, M., Franzetti, M., Simonetti, F. R., Bozzi, G., Binda, F., et al. (2014). Local and global spatio-temporal dynamics of HIV-1 subtype F1. J. Med. Virol. 86, 186-192. doi: 10.1002/jmv.23783

Lai, A., Simonetti, F. R., Zehender, G., De Luca, A., Micheli, V., Meraviglia, P., et al. (2012). HIV-1 subtype F1 epidemiological networks among Italian heterosexual males are associated with introduction events from South America. PLoS One 7:e42223. doi: 10.1371/journal.pone.0042223

Letunic, I., and Bork, P. (2016). Interactive Tree of Life (iTOL) v3: an online tool for the display and annotation of phylogenetic and other trees. Nucleic Acids Res. 44, W242-W245. doi: 10.1093/nar/gkw290

Lole, K. S., Bollinger, R. C., Paranjape, R. S., Gadkari, D., Kulkarni, S. S., Novak, N. G., et al. (1999). Full-length human immunodeficiency virus type 1 genomes from subtype C-infected seroconverters in India, with evidence of intersubtype recombination. J. Virol. 73, 152-160.

Magiorkinis, G., Angelis, K., Mamais, I., Katzourakis, A., Hatzakis, A., Albert, J., et al. (2016). The global spread of HIV-1 subtype B epidemic. Infect. Genet. Evol. 46, 169-179. doi: 10.1016/j.meegid.2016.05.041

Mbisa, J. L., Hué, S., Buckton, A. J., Myers, R. E., Duiculescu, D., Ene, L., et al. (2012). Phylodynamic and phylogeographic patterns of the HIV type 
subtype F1 parenteral epidemic in Romania. AIDS Res. Hum. Retroviruses 2012, 1161-1166. doi: 10.1089/AID.2011.0390

Mehta, S. R., Wertheim, J. O., Delport, W., Ene, L., Tardei, G., Duiculescu, D., et al. (2011). Using phylogeography to characterize the origins of the HIV-1 subtype F epidemic in Romania. Infect. Genet. Evol. 11, 975-979. doi: 10.1016/j.meegid. 2011.03.009

Montano, S. M., Sanchez, J. L., Laguna-Torres, A., Cuchi, P., Avila, M. M., Weissenbacher, M., et al. (2005). Prevalences, genotypes, and risk factors for HIV transmission in South America. J. Acquir. Immune Defic. Syndr. 40, 57-64. doi: 10.1097/01.qai.0000159667.72584.8b

Niculescu, I., Paraschiv, S., Paraskevis, D., Abagiu, A., Batan, I., Banica, L., et al. (2014). Recent HIV-1 outbreak among intravenous drug users in Romania: evidence for cocirculation of CRF14_BG and subtype F1 strains. AIDS Res. Hum. Retroviruses 31, 488-495. doi: 10.1089/aid.2014.0189

Okano, J. T., Robbins, D., Palk, L., Gerstoft, J., Obel, N., and Blower, S. (2016). Testing the hypothesis that treatment can eliminate HIV: a nationwide, population-based study of the Danish HIV epidemic in men who have sex with men. Lancet Infect. Dis. 16, 789-796. doi: 10.1016/S1473-3099(16)30022-6

Op De Coul, E., van den Burg, R., Asjö, B., Goudsmit, J., Cupsa, A., Pascu, R., et al. (2000). Genetic evidence of multiple transmissions of HIV type 1 subtype F within Romania from adult blood donors to children. AIDS Res. Hum. Retroviruses 16, 327-336. doi: 10.1089/088922200309205

Palma, A. C., Araújo, F., Duque, V., Borges, F., Paixão, M. T., Camacho, R., et al. (2007). Molecular epidemiology and prevalence of drug resistance-associated mutations in newly diagnosed HIV-1 patients in Portugal. Infect. Genet. Evol. 7 , 391-398. doi: 10.1016/j.meegid.2007.01.009

Paraschiv, S., Otelea, D., Baicus, C., Tinischi, M., Costache, M., and Neaga, E. (2009). Nucleoside reverse transcriptase inhibitor resistance mutations in subtype F1 strains isolated from heavily treated adolescents in Romania. Int. J. Infect. Dis. 13, 81-89. doi: 10.1016/j.ijid.2008.03.032

Paraskevis, D., Kostaki, E., Beloukas, A., Cañizares, A., Aguilera, A., Rodríguez, J., et al. (2015). Molecular characterization of HIV-1 infection in Northwest Spain (2009-2013): investigation of the subtype F outbreak. Infect. Genet. Evol. 16, 96-101. doi: 10.1016/j.meegid.2014.12.012

Paraskevis, D., Pybus, O., Magiorkinis, G., Hatzakis, A., Wensing, A. M., van de Vijver, D. A., et al. (2009). Tracing the HIV-1 subtype B mobility in Europe: a phylogeographic approach. Retrovirology 6:49. doi: 10.1186/1742-4690-6-49

Peeters, M., D'Arc, M., and Delaporte, E. (2014). The origin and diversity of human retroviruses. AIDS Rev. 16, 23-34.

Pernas, B., Grandal, M., Mena, A., Castro-Iglesias, A., Cañizares, A., Wyles, D. L., et al. (2014). High prevalence of subtype F in newly diagnosed HIV-1 persons in northwest Spain and evidence for impaired treatment response. AIDS 28, 1837-1840. doi: 10.1097/QAD.0000000000000326

Pineda-Peña, A. C., Faria, N. R., Imbrechts, S., Libin, P., Abecasis, A. B., Deforche, K., et al. (2013). Automated subtyping of HIV-1 genetic sequences for clinical and surveillance purposes: performance evaluation of the new REGA version 3 and seven other tools. Infect. Genet. Evol. 19, 337-348. doi: 10.1016/j. meegid.2013.04.032

Pineda-Peña, A. C., Schrooten, Y., Vinken, L., Ferreira, F., Li, G., Trovão, N. S., et al. (2014). Trends and predictors of transmitted drug resistance (TDR) and clusters with TDR in a local Belgian HIV-1 epidemic. PLoS One 9:e101738. doi: 10.1371/journal.pone.0101738

Rambaut, A., Drummond, A. J., Xie, D., Baele, G., and Suchard, M. A. (2018). Posterior summarisation in bayesian phylogenetics using Tracer 1.7. Syst. Biol. 67, 901-904. doi: 10.1093/sysbio/syy032

Rambaut, A., Lam, T. T., Max Carvalho, L., and Pybus, O. G. (2016). Exploring the temporal structure of heterochronous sequences using TempEst (formerly Path-O-Gen). Virus Evol. 2:vew007. doi: 10.1093/ve/vew007

Rambaut, A., Posada, D., Crandall, K. A., and Holmes, E. C. (2004). The causes and consequences of HIV evolution. Nat. Rev. Genet. 5, 52-61. doi: 10.1038/nrg1246

Rodger, A. J., Cambiano, V., Bruun, T., Vernazza, P., Collins, S., van Lunzen, J., et al. (2016). Sexual activity without condoms and risk of HIV transmission in serodifferent couples when the HIV-positive partner is using suppressive antiretroviral therapy. JAMA 316, 171-181. doi: 10.1001/jama.2016. 5148

Rodgers, M. A., Wilkinson, E., Vallari, A., McArthur, C., Sthreshley, L., Brennan, C. A., et al. (2017). Sensitive next-generation sequencing method reveals deep genetic diversity of HIV-1 in the Democratic Republic of the Congo. J. Virol. 91, e1841-e1816. doi: 10.1128/JVI.01841-16
Sayan, M., Willke, A., Ozgunes, N., and Sargin, F. (2013). HIV-1 subtypes and primary antiretroviral resistance mutations in antiretroviral therapy naïve HIV1 infected individuals in Turkey. Jpn. J. Infect. Dis. 66, 306-311. doi: 10.7883/ yoken.66.306

Schultz, A. K., Zhang, M., Bulla, I., Leitner, T., Korber, B., Morgenstern, B., et al. (2009). jpHMM: improving the reliability of recombination prediction in HIV-1. Nucleic Acids Res. 37, W647-W651. doi: 10.1093/nar/ gkp371

Snoeck, J., Van Laethem, K., Hermans, P., Van Wijngaerden, E., Derdelinckx, I., Schrooten, Y., et al. (2004). Rising prevalence of HIV-1 non-B subtypes in Belgium: 1983-2001. J. Acquir. Immune Defic. Syndr. 35, 279-285. doi: 10.1097/ 00126334-200403010-00009

Stadler, T., Kühnert, D., Bonhoeffer, S., and Drummond, A. J. (2013). Birth-death skyline plot reveals temporal changes of epidemic spread in HIV and hepatitis C virus (HCV). Proc. Natl. Acad. Sci. U.S.A. 110, 228-233. doi: 10.1073/pnas. 1207965110

Struck, D., Lawyer, G., Ternes, A. M., Schmit, J. C., and Bercoff, D. P. (2014). COMET: adaptive context-based modeling for ultrafast HIV-1 subtype identification. Nucleic Acids Res. 42:e144. doi: 10.1093/nar/gku739

Suchard, M. A., Lemey, P., Baele, G., Ayres, D. L., Drummond, A. J., and Rambaut, A. (2018). Bayesian phylogenetic and phylodynamic data integration using BEAST 1.10. Virus Evol. 4:vey016. doi: 10.1093/ve/vey016

Tamura, K., Peterson, D., Peterson, N., Stecher, G., Nei, M., and Kumar, S. (2011). MEGA5: molecular evolutionary genetics analysis using maximum likelihood, evolutionary distance, and maximum parsimony methods. Mol. Biol. Evol. 28, 2731-2739. doi: 10.1093/molbev/msr121

Thomson, M. M., Fernández-García, A., Delgado, E., Vega, Y., Díez-Fuertes, F., Sánchez-Martínez, M., et al. (2012). Rapid expansion of a HIV-1 subtype F cluster of recent origin among men who have sex with men in Galicia, Spain. J. Acquir. Immune Defic. Syndr. 59, e49-e51. doi: 10.1097/QAI. ob013e3182400fc4

Triques, K., Bourgeois, A., Saragosti, S., Vidal, N., Mpoudi-Ngole, E., Nzilambi, N., et al. (1999). High diversity of HIV-1 subtype F strains in Central Africa. Virology 259, 99-109. doi: 10.1006/viro.1999.9720

Van Beckhoven, D., Florence, E., Ruelle, J., Deblonde, J., Verhofstede, C., Callens, S., et al. (2015). Good continuum of HIV care in Belgium despite weaknesses in retention and linkage to care among migrants. BMC Infect. Dis. 15:496. doi: 10.1186/s12879-015-1230-3

Vercauteren, J., Derdelinckx, I., Sasse, A., Bogaert, M., Ceunen, H., De Roo, A., et al. (2008). Prevalence and epidemiology of HIV type 1 drug resistance among newly diagnosed therapy-naive patients in Belgium from 2003 to 2006. AIDS Res. Hum. Retroviruses 24, 355-362. doi: 10.1089/aid.2007.0212

Verhofstede, C., Fransen, K., Van Den Heuvel, A., Van Laethem, K., Ruelle, J., Vancutsem, E., et al. (2017). Decision tree for accurate infection timing in individuals newly diagnosed with HIV-1 infection. BMC Infect. Dis. 17:738. doi: 10.1186/s12879-017-2850-6

Vidal, N., Peeters, M., Mulanga-Kabeya, C., Nzilambi, N., Robertson, D., Ilunga, W., et al. (2000). Unprecedented degree of human immunodefiency virus type 1 (HIV-1) group M genetic diversity in the Democratic Republic of Congo suggests that the HIV-1 pandemic originated in Central Africa. J. Virol. 74, 10498-10507. doi: 10.1128/JVI.74.22.10498-10507.2000

Worobey, M., Watts, T. D., McKay, R. A., Suchard, M. A., Granade, T., Teuwen, D. E., et al. (2016). 1970s and 'Patient 0' HIV-1 genomes illuminate early HIV/AIDS history in North America. Nature 539, 98-101. doi: 10.1038/ nature 19827

Conflict of Interest Statement: The authors declare that the research was conducted in the absence of any commercial or financial relationships that could be construed as a potential conflict of interest.

Copyright $\odot 2019$ Vinken, Fransen, Cuypers, Alexiev, Balotta, Debaisieux, SeguinDevaux, García Ribas, Gomes, Incardona, Kaiser, Ruelle, Sayan, Paraschiv, Paredes, Peeters, Sönnerborg, Vancutsem, Vandamme, Van den Wijngaert, Van Ranst, Verhofstede, Stadler, Lemey and Van Laethem. This is an open-access article distributed under the terms of the Creative Commons Attribution License (CC BY). The use, distribution or reproduction in other forums is permitted, provided the original author(s) and the copyright owner(s) are credited and that the original publication in this journal is cited, in accordance with accepted academic practice. No use, distribution or reproduction is permitted which does not comply with these terms. 